\title{
Impact of Dust from Multiple Microenvironments and Diet on PentaBDE Body Burden
}

\author{
Deborah J. Watkins ${ }^{*}, 1$, Michael D. McClean ${ }^{2}$, Alicia J. Fraser ${ }^{3}$, Janice Weinberg ${ }^{4}$, Heather \\ M. Stapleton ${ }^{5}$, Andreas Sjödin ${ }^{6}$, and Thomas F. Webster ${ }^{2}$ \\ ${ }^{1}$ Brown University, Center for Environmental Health and Technology, Providence, RI, USA \\ ${ }^{2}$ Boston University School of Public Health, Dept. of Environmental Health, Boston, MA, USA \\ ${ }^{3}$ Harvard Medical School, Channing Laboratory, Boston, MA, USA \\ ${ }^{4}$ Boston University School of Public Health, Dept. of Biostatistics, Boston, MA, USA \\ ${ }^{5}$ Duke University, Nicholas School of the Environment, Durham, NC, USA \\ ${ }^{6}$ Centers for Disease Control and Prevention (CDC), National Center for Environmental Health \\ (NCEH), Division for Laboratory Sciences (DLS), Atlanta, GA, USA
}

\begin{abstract}
Our objectives were to determine relative contributions of diet and dust exposure from multiple microenvironments to PentaBDE body burden, and to explore the role of handwipes as a measure of personal exposure to PentaBDE. We administered a food frequency questionnaire and collected serum, dust (office, main living area, bedroom, and vehicle) and handwipe samples from 31 participants.
\end{abstract}

$\Sigma$ PentaBDEs (sum of BDE 28/33, 47, 99, 100, and 153) in handwipes collected in the office environment were weakly correlated with dust collected from offices $(r=0.35, p=0.06)$ and bedrooms $(\mathrm{r}=0.39, \mathrm{p}=0.04)$, but not with dust from main living areas $(\mathrm{r}=-0.05, \mathrm{p}=0.77)$ or vehicles $(\mathrm{r}=0.17, \mathrm{p}=0.47)$. $\Sigma$ PentaBDEs in serum were correlated with dust from main living areas $(\mathrm{r}=0.42$, $\mathrm{p}=0.02)$ and bedrooms $(\mathrm{r}=0.49, \mathrm{p}=0.008)$, but not with dust from offices $(\mathrm{r}=0.22, \mathrm{p}=0.25)$ or vehicles $(r=0.20, p=0.41)$. Our final regression model included variables for main living area dust and handwipes, and predicted 55\% of the variation in serum $\Sigma$ PentaBDE concentrations $(\mathrm{p}=0.0004)$. Diet variables were not significant predictors of $\Sigma$ PentaBDEs in serum.

Our research suggests that exposure to dust in the home environment may be the most important factor in predicting PentaBDE body burden in North Americans, and potential exposure pathways may involve PBDE residues on hands.

\section{Introduction}

Polybrominated diphenyl ethers (PBDEs) are brominated flame-retardants that have been widely used in many types of consumer products, and were originally manufactured in three

\footnotetext{
"Corresponding Author: Deborah Watkins, Deborah_Watkins@brown.edu, Brown University, Center for Environmental Health and Technology, 121 South Main Street, Box G-S121-2, Providence, RI 02912, Phone: (401) 863-6274, Fax: (401) 863-3713.

Supporting Information Available

Spearman correlations of $\Sigma$ PentaBDE concentrations in dust between microenvironments; Spearman correlations between individual PentaBDE congeners within each sample type; Mean concentrations of individual PentaBDE congeners in dust and handwipe field blanks; Dietary information including portion size and fat content assumptions, and food (servings/day) as univariate predictors of $\Sigma$ PentaBDEs in serum (ng/g lipid). This information is available free of charge via the Internet at http://pubs.acs.org/.
} 
commercial mixtures: PentaBDE, which was added primarily to polyurethane foam, and OctaBDE and DecaBDE, which were added primarily to plastic polymers used in electronics. In animal studies, exposure to PBDEs has been shown to affect neurodevelopment, reproduction, and disrupt the endocrine system [1]. Human studies have linked PBDE exposure to changes in thyroid and reproductive hormone homeostasis [2-4], neurodevelopment $[5,6]$, fertility [7, 8], and adverse birth outcomes [9, 10].

The PentaBDE and OctaBDE commercial mixtures were voluntarily withdrawn from the United States (US) market by manufacturers and banned in the European Union (EU) in 2004. DecaBDE is currently being phased out in the EU, and manufacturers announced plans to cease use of DecaBDE in the US by the end of 2013 [11]. However, human exposure to these persistent chemicals will likely continue for decades due to continued use of products containing PBDEs and the transfer of such products to landfills where PBDEs leach into the environment and bioaccumulate in the food chain.

We previously reported associations between PBDEs in office dust, handwipes collected in the office environment, and serum [12]. However, little is known about the relative importance of exposures in different microenvironments (i.e. office, home, car). PBDEs are ubiquitous in residential air and dust [13-15], and dust has been shown to be associated with biological measurements of PentaBDEs [16-18]. PBDEs have also been measured in vehicle air and dust [19-21] but it is unknown how exposure in this microenvironment contributes to body burden.

In a review of human exposure to PBDEs, Lorber estimated that in the US approximately 80 to $90 \%$ of PBDE body burden can be attributed to house dust, but there was a great deal of uncertainty in this estimate [22]. Consumption of meat, poultry, and dairy products has also been shown to contribute to PentaBDE body burden [18, 23]. Accordingly, PBDEs in office workers are likely attributable to a combination of diet and exposures to dust at home, the office, and from cars, but the relative contribution of each is unknown. Additionally, our previous work with handwipes suggests that exposure involves PBDEs on hands, either through dermal absorption or incidental ingestion [12]. However, it is unclear if handwipes are a measure of exposure to immediate surroundings or an integrated measure of exposure across several microenvironments.

The main objective of the present study was to determine the relative contributions of dust exposure from homes, offices, and vehicles, as well as diet to PentaBDE body burden. Another, related objective was to further explore the role of handwipes as a measure of personal exposure to PentaBDEs in dust. To address these goals we collected concurrent measures of $\Sigma$ PentaBDEs (sum of BDE 28/33, 47, 99, 100, and 153) in serum, handwipes, and dust from multiple microenvironments and administered a food frequency questionnaire.

\section{Methods}

\section{Study Design}

As previously described elsewhere [12], we recruited a convenience sample of 31 adults who live and work in the Boston, Massachusetts (USA) area. The study population consisted of 26 females and 5 males, was $90 \%$ white, ha d a median age of 49 years, and $65 \%$ owned vehicles. To be eligible for participation, subjects had to work at least 20 hours a week in an office, report being in "good", "very good", or "excellent" health and be a non-smoker. The field effort was conducted from January through March of 2009. Active participation for each subject lasted one week and included the collection of up to four dust samples, a handwipe sample, blood sample, and questionnaire data. The Boston University Medical 
Center institutional review board approved the study protocol and informed consent was obtained from all participants prior to participation.

\section{Dust Samples}

Dust samples were collected from each participant's office, main living area of their home (MLA), bedroom, and vehicle (if applicable) using a standardized protocol. In instances where participants lived in a studio apartment or did not have a separate main living area $(\mathrm{n}=2)$, only one home dust sample was collected. In data analyses, the single dust samples collected from such homes were treated as main living area samples, as the contents of the rooms reflected mixed usage.

Dust samples were collected using a cellulose extraction thimble (Whatman Inc., Piscataway, NJ) inserted between the crevice tool and vacuum tube extender of a Eureka Mighty-Mite vacuum cleaner [14]. To prevent cross-contamination, all equipment used in dust collection and processing was cleaned between samples using a $1 \%$ detergent and hot water solution. Each microenvironment was vacuumed for approximately 10 minutes. In offices, main living areas, and bedrooms, dust was vacuumed from the entire floor surface area of the room including accessible floor space and the tops of immovable furniture. In vehicles, surfaces of both front and back seats were vacuumed. The floor, dashboard, and other surfaces were not vacuumed.

After sample collection, thimbles were wrapped in aluminum foil, sealed in polyurethane bags and stored at room temperature until processed. We collected dust field blanks in randomly selected participant's homes and offices $(n=12)$ by vacuuming sodium sulfate powder (a surrogate for dust) from a clean aluminum foil surface. Dust samples were sieved to collect particles $<500 \mu \mathrm{m}$ in size. Sieved samples were placed in clean amber glass jars and stored at $-20^{\circ} \mathrm{C}$. Dust samples were analyzed for 37 PBDE congeners using gas chromatography-mass spectrometry operated in electron capture negative ionization mode (GC/ECNI-MS), using previously described methods [24].

\section{Handwipe Samples}

Handwipe samples were collected from each participant while they were working in their office environment, typically in the afternoon and at least 60 minutes after they last washed their hands. To collect the handwipe samples, a $3 \mathrm{inch} \times 3$ inch sterile gauze pad was immersed in $3 \mathrm{ml}$ of isopropyl alcohol and used to wipe the palm and back of hand from wrist to fingertips. The handwipe was then placed in a clean glass vial, wrapped in foil and bubble-wrap, and stored at $-20^{\circ} \mathrm{C}$. Left and right hands were sampled separately but both samples were extracted and analyzed together (i.e. one measurement per participant). A field blank wipe sample was paired with the collection of each handwipe by soaking a gauze pad in isopropyl alcohol and placing it directly into the glass vial. Handwipe samples were analyzed for 37 PBDE congeners using GC/ECNI-MS, using previously described methods [24].

\section{Blood Samples}

A phlebotomist collected one $10 \mathrm{ml}$ red top Vacutainer tube of blood from each participant at the end of the workweek. Tubes were allowed to coagulate at room temperature for 1-2 hours and then centrifuged for 15 minutes at $1,000 \times \mathrm{g}$. Serum from each individual was separated into aliquots for analysis of PBDEs $(10 \mathrm{ml}$ amber glass vials) and lipids $(2 \mathrm{ml}$ polypropylene vials) and stored at $-20^{\circ} \mathrm{C}$. Serum samples were analyzed for BDE28, 47, 66, 85, 99, 100, 153, and 154 and lipids at the Centers for Disease Control and Prevention (CDC) using established methods [25]. 


\section{Questionnaire}

Researchers administered a questionnaire to collect information about work and personal habits, including the average number of hours per week participants spend in their offices, if they commute to work by car, and how often they usually wash their hands in a day, as well as personal characteristics such as sex and age.

Dietary information was obtained using a food frequency questionnaire in which participants were asked to report how often they ate a standard portion size of various foods over the past year. Photographs of portion sizes based on USDA guidelines were used to help participants estimate their food intake (portion sizes listed in Supporting Information, Table S1). Participants estimated intake as never, less than once/week, once/week, 2-3 times/week, 4-6 times/week, once/day, or more than once/day. Responses were converted to a linear scale (servings/day) for analysis. Foods such as milk, cheese, beef, chicken, and salmon were analyzed individually as well as in consolidated food groups such as meat, fish, and dairy. The meat variable was comprised of beef, pork, and chicken intake. The fish variable was comprised of tuna and salmon intake. A continuous variable of ounces of dairy fat consumed per day was created by using the industry established fat content for each dairy product to assess overall dairy intake (Supporting Information, Table S1). Dairy products included in this measure were milk (skim, 1\%, whole), cheese (low-fat or regular), yogurt, ice cream, and frozen yogurt. This food frequency questionnaire was also used by Wu et al. in their study of first time mothers [18].

\section{Data Analysis}

Dust samples were corrected by subtracting the mean of the dust field blanks $(\mathrm{n}=12)$; handwipes were corrected by subtracting each individual's paired wipe field blank measurement (dust and handwipe field blank values reported in Supporting Information, Table S2). Serum samples were corrected using the mean of method blanks analyzed in parallel with the samples. Serum limits of detection (LOD) were calculated as three times the standard deviation of method blanks divided by the sample size. Limits of detection for dust and handwipe samples were determined as three times the standard deviation of the appropriate field blanks. In instances where congeners were not detected in the dust and handwipe field blanks, the laboratory instrument detection limit was used as the LOD. Measurements below the LOD were substituted with a value of LOD/2.

For consistency, $\Sigma$ PentaBDE is defined here as the sum of BDE 28/33, 47, 99, 100, and 153, the congeners detected in greater than $50 \%$ of samples within all three media: dust, handwipes, and serum. A sum measure was appropriate as these congeners were highly correlated within each sample type (Supporting Information, Tables S3, S4, S5).

PBDE data were log-normally distributed and natural log-transformed before analysis when appropriate. We used Spearman correlations to explore associations between continuous variables. Regression models were used to evaluate predictors of $\ln$ PBDE concentrations in serum and handwipes. Beta estimates were exponentiated $\left(\mathrm{e}^{\beta}\right)$, producing an estimate of the multiplicative change in outcome. To minimize the effect of skewed data and outliers, categorical variables were created from continuous dust and handwipe data: two-level variables (low, high) were created using the median as a cut point (vehicle dust); three-level variables (low, medium, high) were created using tertiles (main living area, bedroom, and office dust, handwipes). Potential confounders and correlations between independent variables were considered when determining the most parsimonious multivariate model predicting serum $\Sigma$ PentaBDEs, while also considering sample size when determining the number of variables included in models simultaneously. Linear mixed effects models were used to assess differences in dust concentrations across microenvironments while accounting 
for correlation between samples taken from the same home (main living area and bedroom). All statistical analyses were performed using SAS version 9.1 with statistical significance defined as $\mathrm{a}=0.05$.

The blood sample from one participant did not have adequate volume to quantify all PBDE congeners, hence this participant was not included in analyses involving PBDEs in serum, but is included in handwipe analyses. BDE183 and BDE209, the main components of the Octa and Deca formulations, were detected in less than $50 \%$ of serum samples, so these congeners are not included in this analysis.

\section{Results}

Summary statistics for individual PBDE congeners and $\Sigma$ PentaBDEs in dust collected from multiple microenvironments are presented in Table 1. Geometric mean concentrations of $\Sigma$ PentaBDEs in dust from offices, main living areas, bedrooms, and vehicles were 2,170 ng/ $\mathrm{g}, 1,690 \mathrm{ng} / \mathrm{g}, 1,380 \mathrm{ng} / \mathrm{g}$, and 2,610 ng/g respectively. Individual PentaBDE congeners included in the sum measure were detected in 87 to $100 \%$ of all dust samples.

As expected, $\Sigma$ PentaBDE concentrations in dust collected from participant's main living area and bedroom were significantly correlated $(r=0.49, p=0.007)$, but this was the only significant correlation between the four microenvironments (Supporting Information, Table S6). $\Sigma$ PentaBDE concentrations in dust from the four microenvironments were not significantly different from one another $(\mathrm{p}=0.53)$.

Handwipe and serum $\Sigma$ PentaBDE measurements from participants in this study have been presented elsewhere [12]. Briefly, the mass of $\Sigma$ PentaBDEs measured in handwipes ranged from 14 to 2,860 ng with a geometric mean of $70 \mathrm{ng}$. $\Sigma$ PentaBDE concentrations in serum ranged from 3.4 to $348 \mathrm{ng} / \mathrm{g}$ lipid with a geometric mean of $27.7 \mathrm{ng} / \mathrm{g}$ lipid.

\section{Predictors of $\Sigma$ PentaBDEs in Handwipes}

Scatterplots of $\Sigma$ PentaBDEs in dust from the four microenvironments versus $\Sigma$ PentaBDEs in handwipes are shown in Figure 1. Handwipes, which were collected in the office environment, were positively correlated with dust collected from the office $(r=0.35, p=0.06)$, though this result is only marginally significant. $\sum$ PentaBDEs in handwipes were significantly correlated with bedroom dust $(\mathrm{r}=0.39, \mathrm{p}=0.04)$, but not with dust from main living areas $(\mathrm{r}=-0.05, \mathrm{p}=0.77)$ or vehicles $(\mathrm{r}=0.17, \mathrm{p}=0.47)$.

Regression models were used to examine the relationship between categorical variables of $\Sigma$ PentaBDEs in dust from the four microenvironments and $\Sigma$ PentaBDEs in handwipes. The parameter estimates, standard errors, and p- values for these models are presented in Table 2 , with the $\beta$ estimate representing the mean difference in handwipe $\Sigma$ PentaBDEs on the natural-log scale. The results of these models agree with the presented correlations, suggesting associations between handwipes and dust collected from the office and bedroom but not from the main living area or vehicle. As the exponentiated $\beta$ estimate represents the multiplicative change, $\Sigma$ PentaBDEs in handwipes collected from the high office dust group were 3.3 times higher than $\Sigma$ PentaBDEs in handwipes from the low office dust group (3.3 $\left.=\mathrm{e}^{1.2}, \mathrm{p}=0.04\right)$. Similarly, $\Sigma$ PentaBDEs in handwipes collected from the high bedroom dust group were 2.7 times higher than $\Sigma$ PentaBDEs in handwipes from the low bedroom dust group $\left(2.7=\mathrm{e}^{0.98}, \mathrm{p}=0.1\right)$, though this result was not statistically significant. $\Sigma$ PentaBDE concentrations in handwipes collected from the medium dust categories were not significantly different from the low dust categories for either the office $(\mathrm{p}=0.58)$ or the bedroom $(\mathrm{p}=0.99)$. 


\section{Predictors of $\Sigma$ PentaBDEs in Serum}

Scatterplots of $\Sigma$ PentaBDEs in dust from the four microenvironments versus $\Sigma$ PentaBDEs in serum are shown in Figure 2. $\Sigma$ PentaBDEs in serum were significantly correlated with dust from main living areas $(\mathrm{r}=0.42, \mathrm{p}=0.02)$ and bedrooms $(\mathrm{r}=0.49, \mathrm{p}=0.008)$, but not significantly correlated with dust from offices $(\mathrm{r}=0.22, \mathrm{p}=0.25)$ or vehicles $(\mathrm{r}=0.20, \mathrm{p}=0.41)$.

Regression models were used to examine the relationship between categorical variables of $\Sigma$ PentaBDEs in dust from the four microenvironments and $\Sigma$ PentaBDEs in serum. The parameter estimates, standard errors, and p-values for these models are presented in Table 2, with the $\beta$ estimate representing the mean difference in $\Sigma$ PentaBDE concentrations in serum on the natural-log scale. Consistent with scatterplots, $\mathrm{c}$ ategorical $\Sigma$ PentaBDEs in dust from the main living area and bedroom were the strongest, significant predictors of serum $\Sigma$ PentaBDEs. Those in the high and medium main living area dust categories had $3.6\left(\mathrm{e}^{1.29}\right.$, $\mathrm{p}=0.006)$ and $3.8\left(\mathrm{e}^{1.34}, \mathrm{p}=0.003\right)$ times the $\sum$ PentaBDEs in serum compared to those in the low dust category, but were not significantly different from one another $(\mathrm{p}=0.9)$. Similarly, those in the high and medium bedroom dust categories had $3.9\left(\mathrm{e}^{1.37}, \mathrm{p}=0.005\right)$ and 2.2 $\left(\mathrm{e}^{0.81}, \mathrm{p}=0.09\right)$ times the $\Sigma$ PentaBDEs in serum compared to those in the low dust category.

Categories of $\Sigma$ PentaBDEs in vehicle dust were also a significant, albeit weaker predictor of serum $\Sigma$ PentaBDEs, with those in the high vehicle dust category having $2.8\left(\mathrm{e}^{1.04}, \mathrm{p}=0.05\right)$ times the $\Sigma$ PentaBDEs in serum compared to those in the low dust category. This is in contrast to the correlation between $\Sigma$ PentaBDEs in vehicle dust and serum $(\mathrm{r}=0.20, \mathrm{p}=0.41)$, which did not suggest a significant association (Figure 2). $\Sigma$ PentaBDEs in office dust were not a significant predictor of $\Sigma$ PentaBDEs in serum $(\mathrm{p}=0.27)$.

Dietary factors were not significant predictors of $\Sigma$ PentaBDEs in serum, regardless of whether they were evaluated as continuous or categorical variables (Supporting Information, Table S7).

\section{Multivariate Model Predicting $\Sigma$ PentaBDEs in Serum}

Although no diet variables predicted $\Sigma$ PentaBDEs in serum, they were included one at a time in models with dust variables to ensure that the relationship between $\Sigma$ PentaBDEs in dust and serum was not confounded by diet. There was no evidence of confounding such that diet variables were excluded from final models. Vehicle dust was a significant predictor of $\Sigma$ PentaBDEs in serum but was not included in multivariate models as this would reduce our sample size by a third, limiting our statistical power.

The first multivariate regression model (model 1) included dust from all three indoor microenvironments (main living area, bedroom, and office). The parameter estimates, standard errors, and p-values of model 1 are presented in Table 3 . The overall model was statistically significant $(\mathrm{p}=0.04$ ) and explained $45 \%$ of the variation in serum $\Sigma$ PentaBDEs, but individual variables were not significant. $\Sigma$ PentaBDEs in main living area and bedroom dust represent exposure to $\Sigma$ PentaBDEs in the home environment and predicted serum $\Sigma$ PentaBDEs in univariate models, but they were also moderately correlated with one another. The results of model 1 suggest that including these two exposure measures together may not be the most parsimonious model in predicting $\Sigma$ PentaBDEs in serum.

We previously reported that $\Sigma$ PentaBDEs in handwipes were a predictor of $\Sigma$ PentaBDEs in serum $(r=0.44, p=0.01)$ [12], therefore we included handwipes in two different multivariate regression models, one with main living area dust and one with bedroom dust. Main living area dust and handwipes explained a larger portion of variability of $\Sigma$ PentaBDEs in serum $\left(\mathrm{R}^{2}=0.55\right)$ compared to the model with bedroom dust and handwipes $\left(\mathrm{R}^{2}=0.38\right)$. As bedroom dust and handwipes were moderately correlated $(r=0.39)$, they may partially be a measure 
of exposure from the same microenvironment. Taking these factors into consideration, the final model (model 2) included main living area dust to reflect exposure in the home, and handwipes to reflect exposure in the office. The parameter estimates, standard errors, and pvalues for model 2 are shown in Table 3.

The overall model 2 was statistically significant $(\mathrm{p}=0.0004)$ and predicted $55 \%$ of the variation in serum $\Sigma$ PentaBDEs. Main living area dust was a significant predictor of $\sum$ PentaBDEs in serum $(\mathrm{p}=0.001)$, with those in the high category having $3.7\left(\mathrm{e}^{1.31}\right)$ times the $\Sigma$ PentaBDEs in their serum compared to those in the low category $(\mathrm{p}=0.002)$, and those in the medium category having $4.1\left(\mathrm{e}^{1.40}\right)$ times the $\Sigma$ PentaBDEs in their serum compared to the low category $(\mathrm{p}=0.0006)$. However, $\Sigma$ PentaBDEs in serum were not statistically different between the high and medium main living area dust categories $(\mathrm{p}=0.8)$. Handwipes were also statistically significant predictors of $\Sigma$ PentaBDEs in serum ( $\mathrm{p}=0.007$ ), with those in the high handwipe category having $3.0\left(\mathrm{e}^{1.11}\right)$ times the PentaBDEs in their serum compared to the low handwipe category $(\mathrm{p}=0.005)$, and those in the medium category having $2.9\left(\mathrm{e}^{1.07}\right)$ times the $\Sigma$ PentaBDEs in their serum compared to the low handwipe category $(\mathrm{p}=0.007)$.

\section{Discussion}

Previous studies have shown associations between house dust and biological measures of PentaBDEs [16-18], but little is known about the biological relevance of exposure in offices and vehicles. This is the first comprehensive study of exposure to PentaBDEs across multiple microenvironments. By collecting dust samples from the home, office, and vehicle of each participant, paired with a serum sample, we were able to examine the relative contribution of dust exposure in these microenvironments to PentaBDE body burden. Diet has also been associated with biological measures of PentaBDEs [18, 23], but has never been evaluated concurrently with dust exposure from multiple microenvironments. Administering a food frequency questionnaire allowed us to simultaneously examine exposure via indoor dust and diet, the two main exposure pathways thought to contribute to PentaBDE body burdens in the US.

\section{Exposure in the home}

$\Sigma$ PentaBDE concentrations in dust from main living areas and bedrooms were the strongest predictors of $\Sigma$ PentaBDEs in serum, suggesting that exposure to dust in the home might be the most important contributor to PBDE body burden. Although geometric mean concentrations of $\Sigma$ PentaBDEs in office and vehicle dust were higher than those measured in homes, differences in behavior and the amount of time spent in each microenvironment likely explains the larger contribution of the home environment to PentaBDE body burden. For example, behavior in offices is usually limited to sitting at a desk with a computer, whereas behavior in the home varies greatly and includes activities such as cleaning, sitting/ laying on older foam furniture, or playing on the floor with children, which likely results in greater contact with dust.

The association between handwipes and serum suggests that people are exposed to PentaBDEs via hands, either by hand to mouth activity or dermal absorption [12].

Handwipes collected in participant's offices were associated with $\Sigma$ PentaBDEs in dust from both offices and bedrooms. The association with office dust suggests that handwipes reflect recent exposure to the surrounding environment, but the association with bedroom dust is less clear. One possible explanation is that handwipes are an integrated measure of exposure from the immediate environment as well as environment over the past several hours. More research is needed to fully characterize exposure captured by handwipes, and what other factors play a role in levels of PentaBDEs on hands. 


\section{Comparisons with other studies}

Measurements of $\Sigma$ PentaBDEs in handwipes and serum were lower than those previously reported in our handwipe pilot study [24] and in NHANES serum samples [26], respectively. In both instances, samples were collected years prior to those collected in this study. During this time PentaBDEs were phased-out of use in new consumer products in the US, potentially explaining this apparent decrease in exposure. However, as our sample size is small, the decrease could also be due to chance. Our reported concentrations of $\Sigma$ PentaBDEs in office dust are higher than in offices in Europe and Asia [27-31], but similar to measures reported elsewhere in the US [32].

Several studies have reported PentaBDE concentrations in home dust, but variations in collection methods make comparisons difficult. In 2006, Allen et al. studied a similar population in the Boston area, using the same collection methods and analytical laboratory as that used in the current study [14]. We found lower concentrations of $\Sigma$ PentaBDEs in main living area dust and bedroom dust than those reported by Allen et al. - approximately $60 \%$ lower in main living area dust and $40 \%$ lower in bedroom dust. Again, PentaBDEs were phased-out in 2004, so presumably some products containing PentaBDEs were taken out of use in the time between the two studies. However, as sample sizes in both studies were relatively small, the apparent decrease could also be due to chance. Concentrations of $\Sigma$ PentaBDEs in vehicle dust $(2,610 \mathrm{ng} / \mathrm{g})$ were similar to those reported by Lagalante et al. in $2008(2,502 \mathrm{ng} / \mathrm{g})[19]$.

A few US studies have previously examined associations between PentaBDEs in house dust and biological measures of PentaBDE body burden. Johnson et al. reported a geometric mean concentration of $\Sigma$ PentaBDEs in serum (29.8 ng/g lipid) similar to that reported here (27.8 ng/g lipid) [17]. They also report correlations of $0.65-0.89$ between PentaBDE congeners in serum and vacuum bag dust. Wu et al. found a correlation of 0.64 between $\Sigma$ PentaBDEs in house dust and breast milk collected from first-time mothers [18], a biological measurement equivalent to serum when PBDE concentrations are lipidnormalized [33]. In comparison, we observed smaller correlations between serum $\Sigma$ PentaBDEs and researcher collected dust from the home (MLA: $r=0.42$, bedroom: $r=0.49$ ). This difference between studies could be due to several factors, including differences in dust collection and processing methods, differences in study populations, or timing of sample collection.

Although previous studies have reported associations between diet and PentaBDE body burden, we did not observe this association in our study population. Wu et al. used the same food frequency questionnaire as was used in this study and found associations between PBDEs in breast milk and reported dairy fat and meat intake [18]. Fraser et al. also observed an association between serum PentaBDE concentrations and beef and poultry intake using NHANES data from 2003/2004 [23]. One possible explanation for our inability to detect a similar association is our small sample size. In addition, Wu et al. studied first-time mothers, asking about pre-pregnancy diet, while our population was predominantly older women working in offices. It is possible that first-time mothers may report or recall their diet differently, and may eat differently than participants in the current study. We found that although on average consumption was similar in the two populations, first-time mothers ate slightly more meat and dairy, and intake was more varied compared to our study population. Another possible explanation is timing, as $\mathrm{Wu}$ et al. collected samples five years prior to this study. As mentioned previously, during this period PentaBDEs were phased-out of production in the US. There is some evidence that during this time PentaBDE concentrations in food have decreased, as Huwe and West recently reported a 63\% decrease of the most prevalent PBDE congeners in meat and poultry samples between 2002 and 2008 [34]. The potential decrease of PentaBDEs in food as well as our inability to detect an association 
between diet and PentaBDE body burden could suggest that diet as a source of PentaBDE exposure is declining, but more research is necessary to confirm this. In addition, exposure to PBDEs through diet is likely misclassified due to the inherent limitations of food frequency questionnaires [35], reducing our ability to detect an association.

\section{Limitations}

Our small sample size limited the number of variables we were able to evaluate simultaneously, as well as our ability to detect weak associations and address potential confounding. Our population was also a relatively homogeneous sample, with most participants being white, older, well-educated women who work in offices in the US. This may limit the generalizability of this study to other populations but shouldn't affect the internal validity of our study.

Our cross-sectional design could also be a limitation, as we collected one dust sample from each microenvironment, one handwipe, and one serum sample from each participant at one point in time. This has different implications depending on sample type. Allen et al. found that $\Sigma$ PentaBDE concentrations in residential dust were strongly correlated over an 8-month period [14], and we would expect this to hold true for the homes sampled in this study, as well as for offices and vehicles. PentaBDE congeners are estimated to have biological halflives on the order of years [36], so serum concentrations of these compounds should reflect long-term exposure. In contrast, handwipe samples presumably reflect recent exposure and are likely influenced by the timing of collection. For example, we previously reported that a dichotomous measure of self-reported hand 4 washing ( $<4$ vs. $\geq 4$ times per day) was a significant predictor of $\Sigma$ PentaBDEs in handwipes, although the amount of time between handwipe collection and last previous handwashing was not [12]. Multiple handwipe samples collected over time in different microenvironments would have helped us better characterize total exposure via this pathway.

Lastly, as the method of sampling dust that is most relevant to exposure is still unclear, misclassification of exposure to $\Sigma$ PentaBDEs in dust is possible, which would most-likely bias our correlation results towards the null. However, as all microenvironments examined in this study were sampled in similar ways, dust collection methods should not influence the relative contributions of the different microenvironments to $\Sigma$ PentaBDE body burden, or our conclusion that the home environment may be the most important microenvironment for exposure.

Our research suggests that exposure to dust in the home environment may be the most important factor in predicting PentaBDE body burden. The relative contribution of exposure through diet is still unclear, as although previous studies observed an association between food intake and PentaBDE body burden, no such association was seen in this study. Our work also suggests that as PentaBDEs are no longer manufactured in the US or used in new consumer products, there may have been a decrease of PentaBDEs in the indoor environment, as well as in food.

\section{Supplementary Material}

Refer to Web version on PubMed Central for supplementary material.

\section{Acknowledgments}

We thank the study participants, Stephanie Chan, Dr. Jessica Nelson, Heather Simpson, Jennifer Valerio, and Courtney Walker. This work was supported by R01ES015829 and T32ES014562 from the National Institute of Environmental Health Sciences. 


\section{References}

1. Birnbaum LS, Staskal DF. Brominated flame retardants: Cause for concern? Environ. Health Perspect. 2004; 112:9-17. [PubMed: 14698924]

2. Meeker JD, Johnson PI, Camann D, Hauser R. Polybrominated diphenyl ether (PBDE) concentrations in house dust are related to hormone levels in men. Sci. Total Environ. 2009; 407:3425-3429. [PubMed: 19211133]

3. Chevrier J, Harley KG, Bradman A, Gharbi M, Sjödin A, Eskenazi B. Polybrominated diphenyl ether (PBDE) flame retardants and thyroid hormone during pregnancy. Environ. Health Perspect. 2010; 118:1444-1449. [PubMed: 20562054]

4. Turyk ME, Persky VW, Imm P, Knobeloch L, Chatterton R, Anderson HA. Hormone disruption by PBDEs in adult male sport fish consumers. Environ. Health Perspect. 2008; 116:1635-1641. [PubMed: 19079713]

5. Herbstman JB, Sjödin A, Kurzon M, Lederman SA, Jones RS, Rauh V, Needham LL, Tang D, Niedzwiecki M, Wang RY, Perera F. Prenatal Exposure to PBDEs and neurodevelopment. Environ. Health Perspect. 2010; 118:712-719. [PubMed: 20056561]

6. Roze E, Meijer L, Bakker A, Van Braeckel KN, Sauer PJ, Bos AF. Prenatal exposure to organohalogens, including brominated flame retardants, influences motor, cognitive, and behavioral performance at school age. Environ. Health Perspect. 2009; 117:1953-1958. [PubMed: 20049217]

7. Akutsu K, Takatori S, Nozawa S, Yoshiike M, Nakazawa H, Hayakawa K, Makino T, Iwamoto T. Polybrominated diphenyl ethers in human serum and sperm quality. Bull. Environ. Contam. Toxicol. 2008; 80:345-350. [PubMed: 18320132]

8. Harley KG, Marks AR, Chevrier J, Bradman A, Sjödin A, Eskenazi B. PBDE concentrations in women's serum and fecundability. Environ. Health Perspect. 2010; 118:699-704. [PubMed: 20103495]

9. Chao H-R, Wang S-L, Lee W-J, Wang Y-F, Papke O. Levels of polybrominated diphenyl ethers (PBDEs) in breast milk from central Taiwan and their relation to infant birth outcome and maternal menstruation effects. Environ. Int. 2007; 33:239. [PubMed: 17079016]

10. Main KM, Kiviranta H, Virtanen HE, Sundqvist E, Tuomisto JT, Tuomisto J, Vartiainen T, Skakkebaek NE, Toppari J. Flame retardants in placenta and breast milk and cryptorchidism in newborn boys. Environ. Health Perspect. 2007; 115:1519-1526. [PubMed: 17938745]

11. U.S. Environmental Protection Agency. Washington, DC: 2010. DecaBDE Phase-out Initiative. http://www.epa.gov/oppt/existingchemicals/pubs/actionplans/deccadbe.html

12. Watkins D, McClean MD, Fraser AJ, Weinberg J, Stapleton HM, Sjödin A, Webster TF. Exposure to PBDEs in the office environment: Evaluating the relationship between dust, handwipes and serum. Environ. Health Perspect. 2011; 119:1247-1252. [PubMed: 21715243]

13. Allen JG, McClean MD, Stapleton HM, Nelson JW, Webster TF. Personal exposure to polybrominated diphenyl ethers (PBDEs) in residential indoor air. Environ. Sci. Technol. 2007; 41:4574-4579. [PubMed: 17695899]

14. Allen JG, McClean MD, Stapleton HM, Webster TF. Critical factors in assessing exposure to PBDEs via house dust. Environ. Int. 2008; 34:1085-1091. [PubMed: 18456330]

15. Harrad S, de Wit CA, Abdallah MA, Bergh C, Bjorklund JA, Covaci A, Darnerud PO, de Boer J, Diamond M, Huber S, Leonards P, Mandalakis M, Ostman C, Haug LS, Thomsen C, Webster TF. Indoor contamination with hexabromocyclododecanes, polybrominated diphenyl ethers, and perfluoroalkyl compounds: An important exposure pathway for people? Environ. Sci. Technol. 2010; 44:3221-3231. [PubMed: 20387882]

16. Frederiksen M, Thomsen C, Froshaug M, Vorkamp K, Thomsen M, Becher G, Knudsen LE. Polybrominated diphenyl ethers in paired samples of maternal and umbilical cord blood plasma and associations with house dust in a Danish cohort. Int. J. Hyg. Environ. Health. 2010; 213:233242. [PubMed: 20471317]

17. Johnson PI, Stapleton HM, Sjödin A, Meeker JD. Relationships between polybrominated diphenyl ether concentrations in house dust and serum. Environ. Sci. Technol. 2010; 44:5627-5632.

[PubMed: 20521814] 
18. Wu N, Herrmann T, Paepke O, Tickner J, Hale R, Harvey LE, La Guardia M, McClean MD, Webster TF. Human exposure to PBDEs: Associations of PBDE body burdens with food consumption and house dust concentrations. Environ. Sci. Technol. 2007; 41:1584-1589. [PubMed: 17396645]

19. Lagalante AF, Oswald TD, Calvosa FC. Polybrominated diphenyl ether (PBDE) levels in dust from previously owned automobiles at United States dealerships. Environ. Int. 2009; 35:539-544. [PubMed: 19019437]

20. Mandalakis M, Stephanou EG, Horii Y, Kannan K. Emerging contaminants in car interiors: Evaluating the impact of airborne PBDEs and PBDD/Fs. Environ. Sci. Technol. 2008; 42:64316436. [PubMed: 18800511]

21. Harrad S, Hazrati S, Ibarra C. Concentrations of polychlorinated biphenyls in indoor air and polybrominated diphenyl ethers in indoor air and dust in Birmingham, United Kingdom: Implications for human exposure. Environ. Sci. Technol. 2006; 40:4633-4638. [PubMed: 16913117]

22. Lorber M. Exposure of Americans to polybrominated diphenyl ethers. J. Expo. Sci. Environ. Epidemiol. 2008; 18:2-19. [PubMed: 17426733]

23. Fraser AJ, Webster TF, McClean MD. Diet contributes significantly to the body burden of PBDEs in the general U.S. population. Environ. Health Perspect. 2009; 117:1520-1525. [PubMed: 20019900]

24. Stapleton HM, Kelly SM, Allen JG, McClean MD, Webster TF. Measurement of polybrominated diphenyl ethers on hand wipes: Estimating exposure from hand-to-mouth contact. Environ. Sci. Technol. 2008; 42:3329-3334. [PubMed: 18522114]

25. Sjödin A, Jones RS, Focant JF, Lapeza C, Wang RY, McGahee EE 3rd, Zhang Y, Turner WE, Slazyk B, Needham LL, Patterson DG Jr. Retrospective time-trend study of polybrominated diphenyl ether and polybrominated and polychlorinated biphenyl levels in human serum from the United States. Environ. Health Perspect. 2004; 112:654-658. [PubMed: 15121506]

26. Sjödin A, Wong L-Y, Jones RS, Park A, Zhang Y, Hodge C, Dipietro E, McClure C, Turner W, Needham LL, Patterson DG. Serum concentrations of polybrominated diphenyl ethers (PBDEs) and polybrominated biphenyl (PBB) in the United States population: 2003-2004. Environ. Sci. Technol. 2008; 42:1377-1384. [PubMed: 18351120]

27. Suzuki G, Nose K, Takigami H, Takahashi S, Sakai S. PBDEs and PBDD/Fs in house and office dust from Japan. Organohalogen Compd. 2006; 68:1843-1846.

28. Huang Y, Chen L, Peng X, Xu Z, Ye Z. PBDEs in indoor dust in South-Central China: Characteristics and implications. Chemosphere. 2010; 78:169-174. [PubMed: 19875146]

29. Roosens L, Cornelis C, D'Hollander W, Bervoets L, Reynders H, Van Campenhout K, Van Den Heuvel R, Neels H, Covaci A. Exposure of the Flemish population to brominated flame retardants: Model and risk assessment. Environ. Int. 2010; 36:368-376. [PubMed: 20226530]

30. Toms LM, Bartkow ME, Symons R, Paepke O, Mueller JF. Assessment of polybrominated diphenyl ethers (PBDEs) in samples collected from indoor environments in South East Queensland, Australia. Chemosphere. 2009; 76:173-178. [PubMed: 19394067]

31. Harrad S, Ibarra C, Abdallah MA-E, Boon R, Neels H, Covaci A. Concentrations of brominated flame retardants in dust from United Kingdom cars, homes, and offices: Causes of variability and implications for human exposure. Environ. Int. 2008; 34:1170-1175. [PubMed: 18558431]

32. Batterman S, Godwin C, Chernyak S, Jia C, Charles S. Brominated flame retardants in offices in Michigan, USA. Environ. Int. 2010; 36:548-556. [PubMed: 20483456]

33. Schecter A, Colacino J, Sjödin A, Needham L, Birnbaum L. Partitioning of polybrominated diphenyl ethers (PBDEs) in serum and milk from the same mothers. Chemosphere. 2010; 78:1279-1284. [PubMed: 20079522]

34. Huwe JK, West M. Polybrominated diphenyl ethers in U.S. meat and poultry from two statistically designed surveys showing trends and levels from 2002 to 2008. J. Agric. Food Chem. 2011

35. Kipnis V, Midthune D, Freedman L, Bingham S, Day NE, Riboli E, Ferrari P, Carroll RJ. Bias in dietary-report instruments and its implications for nutritional epidemiology. Public Health Nutrition. 2002; 5:915-923. [PubMed: 12633516] 
36. Geyer HJ, Schramm KJ, Darnerud PO, Aune M, Feicht EA, Fried KW, Henkelmann B, Lenoir D, Schmid P, McDonald TA. Terminal elimination half-lives of the brominated flame retardants TBBPA, HBCD, and lower brominated PBDEs in humans. Organohalogen Compd. 2004; 66:3867-3872. 
Office Dust vs. Handwipes ( $\mathbf{n}=\mathbf{3 1})$

Bedroom Dust vs. Handwipes $(n=29)$
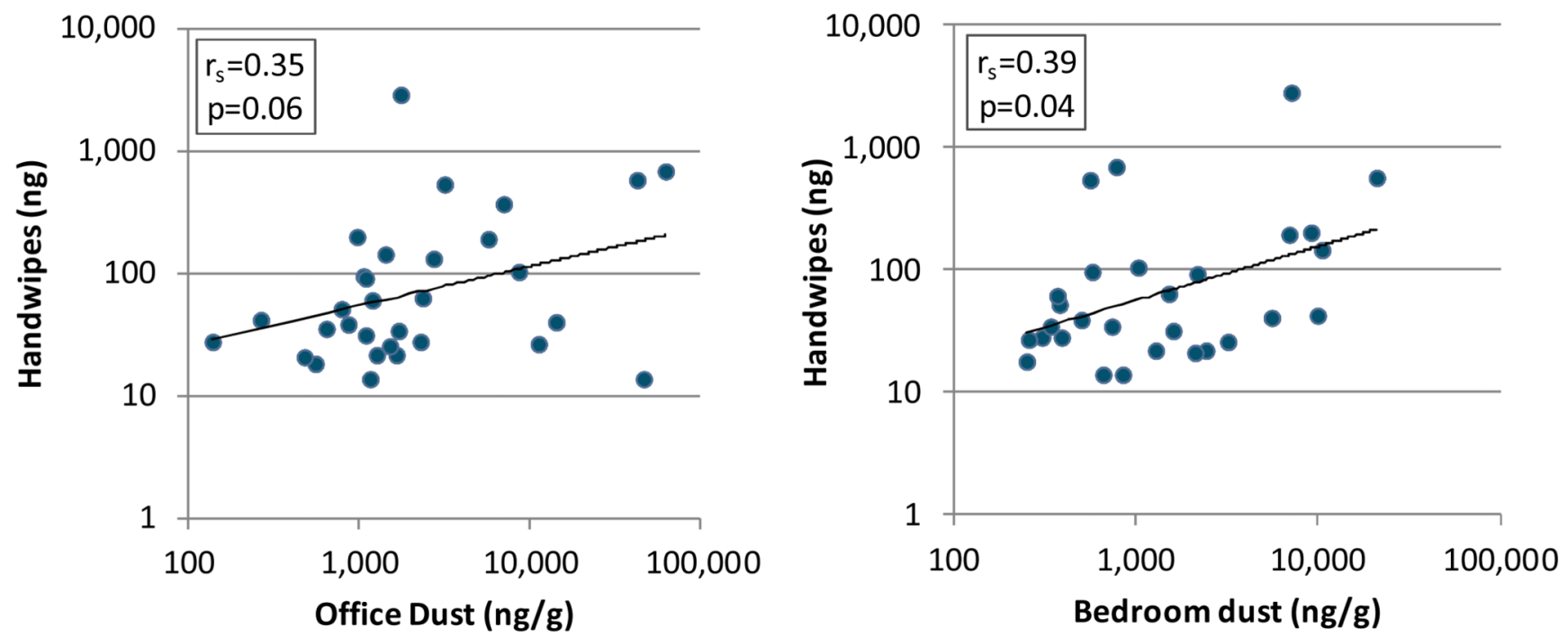

MLA Dust vs. Handwipes $(n=31)$

Vehicle Dust vs. Handwipes $(n=20)$
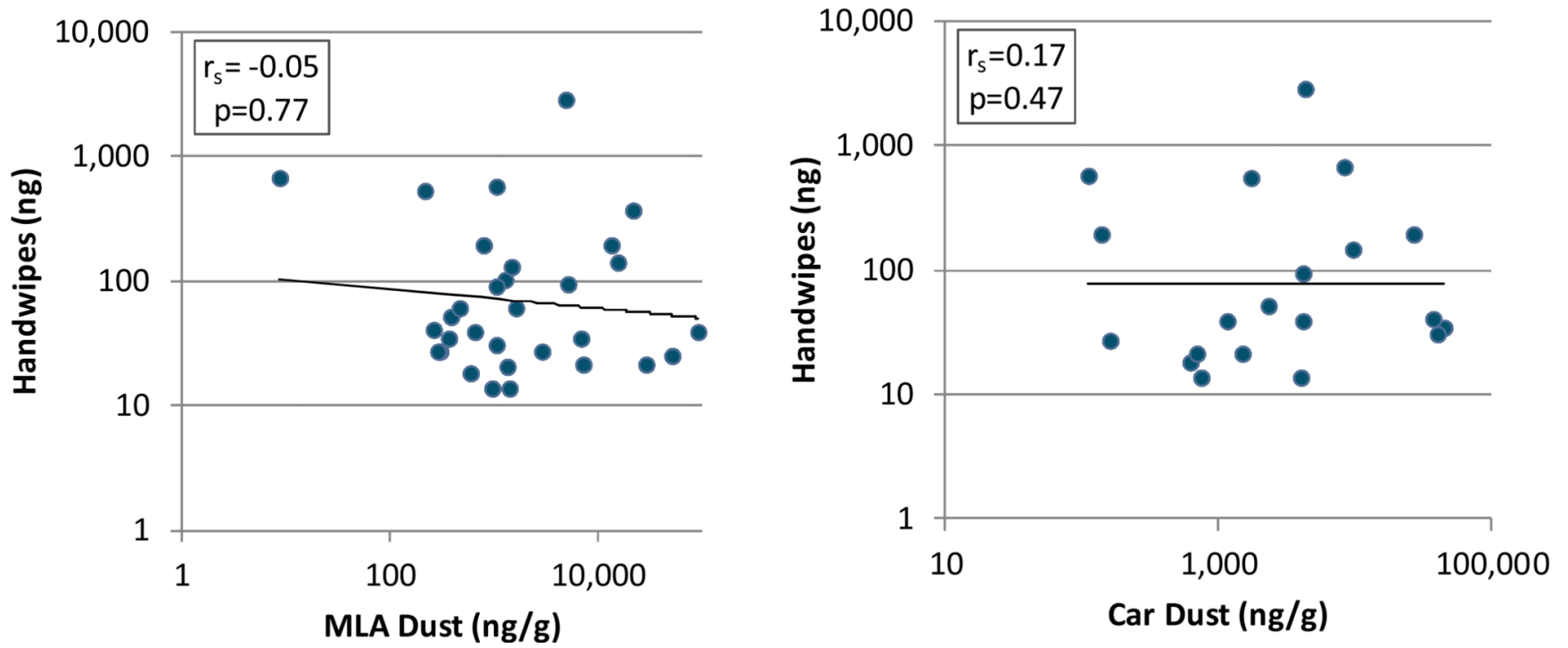

Figure 1.

Correlations of $\Sigma$ PentaBDEs in dust from four microenvironments with $\Sigma$ PentaBDEs in handwipes

$\mathrm{r}_{\mathrm{s}}=$ Spearman correlation coefficient 
Office Dust vs. Serum $(n=30)$

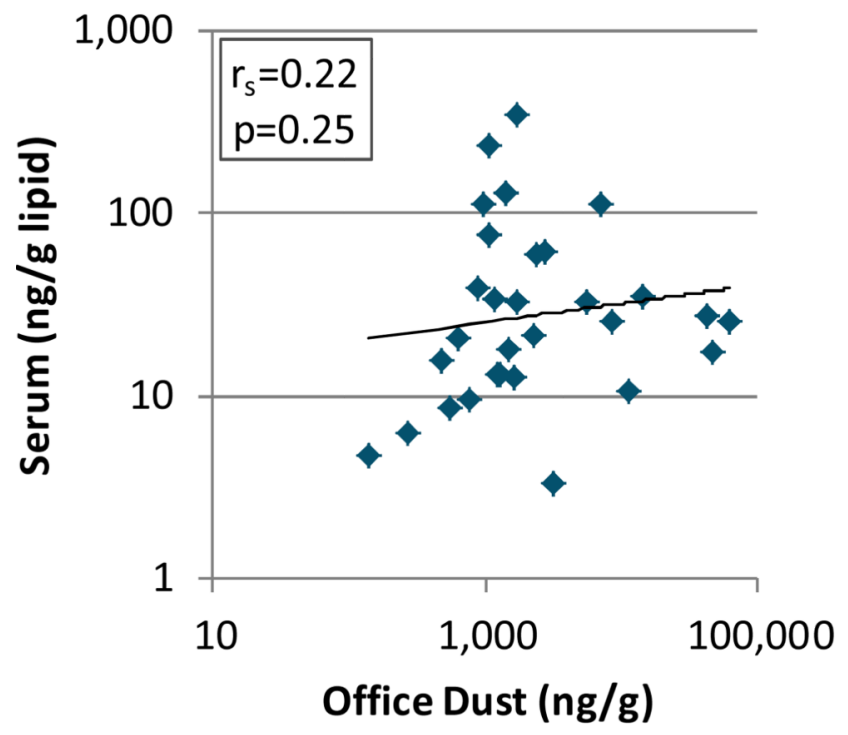

MLA Dust vs. Serum $(n=30)$

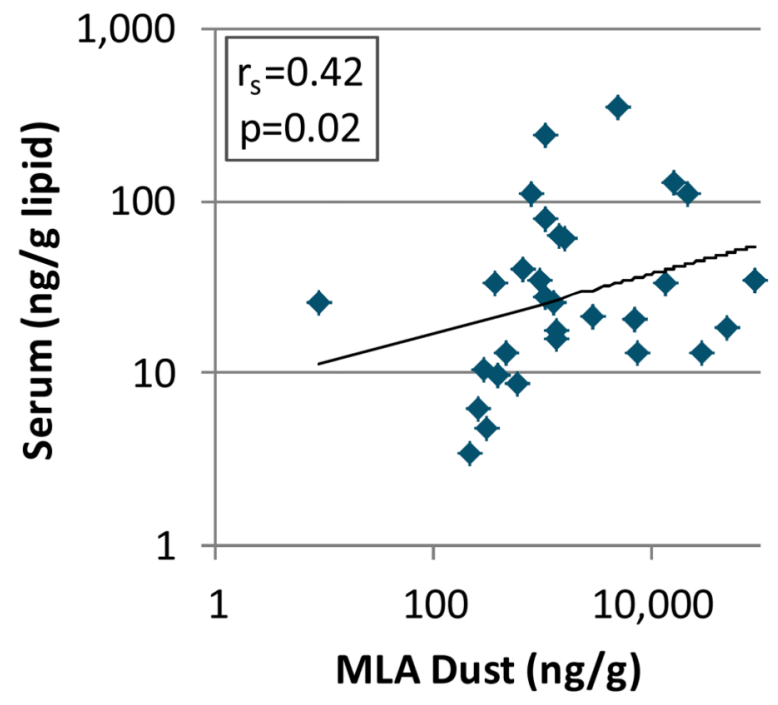

Bedroom Dust vs. Serum $(\mathbf{n}=\mathbf{2 8})$

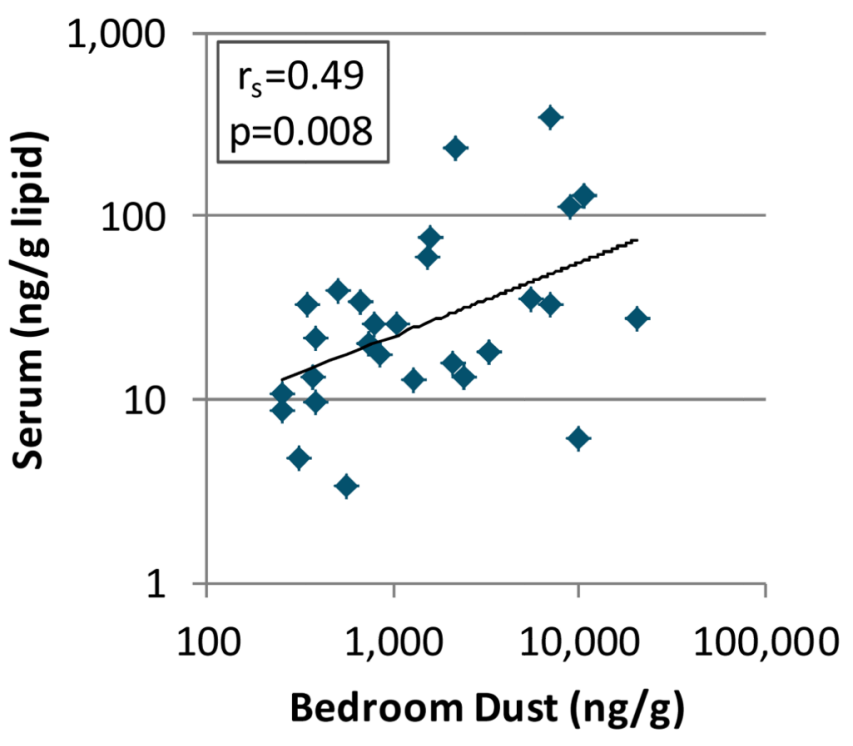

Vehicle Dust vs. Serum $(\mathbf{n}=\mathbf{2 0})$

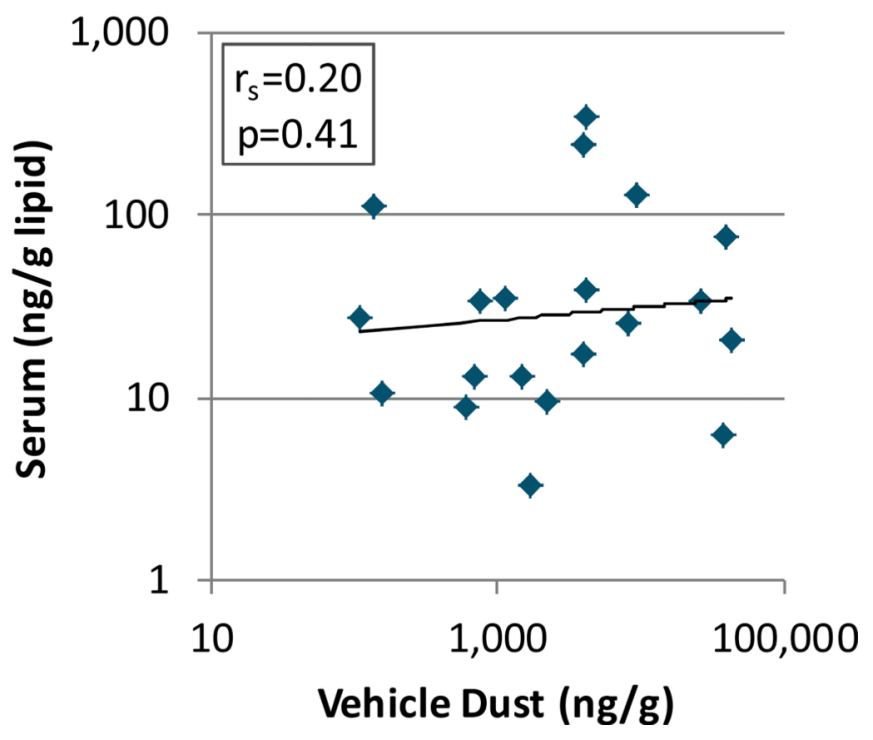

Figure 2.

Correlations of $\Sigma$ PentaBDEs in dust from four microenvironments with $\Sigma$ PentaBDEs in serum

$\mathrm{r}_{\mathrm{s}}=$ Spearman correlation coefficient 


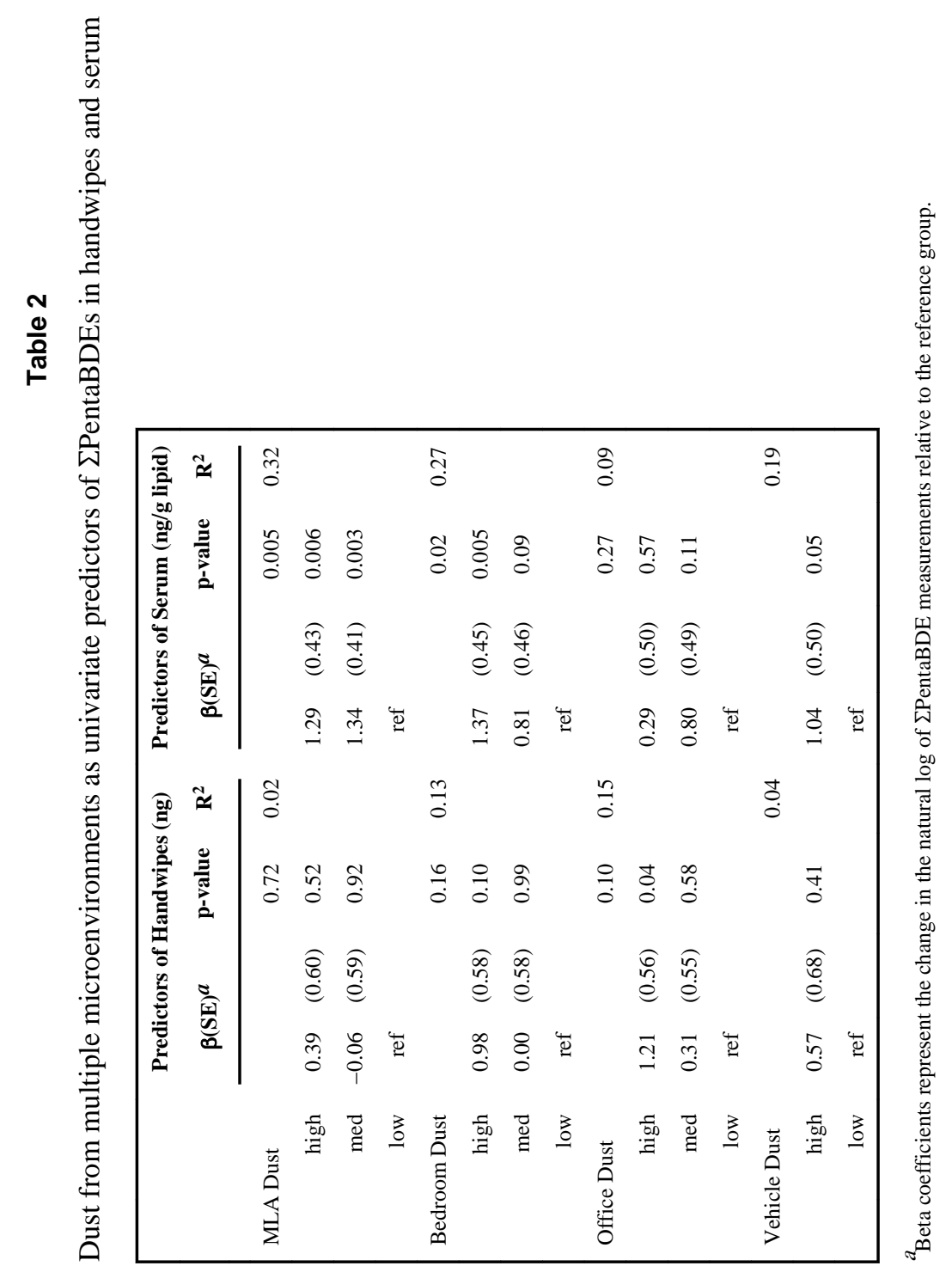

Environ Sci Technol. Author manuscript; available in PMC 2013 January 17. 
